\title{
Awareness of Consequences in Agenesis of the Corpus Callosum: Semantic Analysis of Responses
}

\begin{abstract}
Objective: Agenesis of the corpus callosum (AgCC) in individuals with general intelligence within the normal range results in a syndrome of mild to moderate deficiencies in cognitive, emotional, and social functioning that are still being explored. Anecdotal accounts from families suggest that these cognitive and psychosocial deficiencies affect the ability of these individuals to anticipate the consequences of their decisions and behaviors. This research was designed to clarify the nature of social and emotional cognition in $\mathrm{AgCC}$ with respect to imagination of the consequences of decisions by assessing responses from the Awareness of Consequences Scale (AOCS). Method: Verbal AOCS responses from persons with $\mathrm{AgCC}$ and age and IQ-matched neurotypical controls were scored in the normal manner, and also subjected to semantic analyses using both Latent Semantic Analysis and Linguistic Inquiry and Word Count. Results: It was found that, relative to neurotypical controls, individuals with AgCC scored significantly lower on the typical scoring of the AOCS, had non-typical semantic content in their responses, and used fewer emotion and cognitive content (insight) words. These results were apparent in responses to the three most complex of the AOCS scenarios.
\end{abstract}

Conclusions: Results were consistent with the hypothesis that persons with AgCC are deficient in the capacity to imagine the emotional and cognitive consequences of potential actions on others. particularly in the face of greater situational and social complexity. 
Keywords: Callosal agenesis, action consequences, inferences of emotion, semantic analysis

Public Significance: This research contributes to understanding the life consequences of a thus far poorly studied congenital brain disorder, agenesis of the corpus callosum. It shows that persons with this disorder have trouble realizing the consequences of their decisions and behaviors in complex social situations. 


\section{Introduction}

Agenesis of the corpus callosum (AgCC) is a condition in which the corpus callosum fails to develop, or only partially develops, in utero (Paul, Brown, Adolphs et al., 2007; Shevell, 2002). Although AgCC has often been associated with significant mental deficiencies, it also occurs in individuals with normal intelligence (known as primary $\mathrm{AgCC}$ ). The syndrome associated with primary AgCC appears to involve mild to moderate deficits in cognitive, emotional, and social

functioning. However, the specific nature of these deficiencies in still somewhat unclear. To better understand the nature of their capacity to imagine social and emotional consequences of potential action decisions, we studied responses of persons with $\mathrm{AgCC}$ and controls on the Awareness of Consequences Scale (AOCS; Schwartz, 1968a) using a subjective scoring system and two methods of semantic analysis: Latent Semantic Analysis (LSA; Landauer, 2997) and Linguistic Word and Inquiry Count (LIWC; Pennebaker, Francis, \& Booth, 2001).

\section{Callosal Agenesis}

The corpus callosum consists of over 190,000,000 axons that make both homotopic and heterotopic connections between the two hemispheres. Failure of prenatal development in AcCC can result in either complete or partial callosal absence (Rauch and Jinkins, 1994). Morphological abnormalities in the brain shown to be consistent with callosal agenesis are displacement of the lateral and third ventricles, selective dilation of the posterior horns, and abnormal orientation of gyral markings (Shevell, 2002).

The prevalence of $\mathrm{AgCC}$ (both partial and complete) has been estimated to be 1 in 4000 in the general population (Glass, Shaw, Ma, \& Sherr, 2008; Guillem, Fabre, Cans, Robert-Gnansia, \& Jouk, 2003; Paul et al., 2007; Wang, Huang, \& Yeh, 2004). The underlying cause has been identified in slightly less than half of AgCC cases (Shevell, 2002; Paul et al., 2007). When identified, etiology 
may be related to chromosomal abnormalities, metabolic disorders, and genetic abnormalities (Shevell, 2002; Paul et al., 2007). Though AgCC has been frequently associated with mental retardation, it also occurs in high functioning, neurologically asymptomatic individuals with normalrange IQ scores (Chiarello, 1980; Sauerwein, Nolin, \& Lassonde, 1994). Because these individuals have few, if any, other significant brain abnormalities, they are sometimes referred to as having primary $\mathrm{AgCC}$ since the cognitive outcome is primarily related to callosal agenesis (Brown, Paul, Symington, \& Dietrich, 2005a).

The syndrome associated with primary $\mathrm{AgCC}$ has been characterized thus far as involving normal full-scale intelligence, but with mild to moderate deficits in specific domains of cognitive ability (Brown \& Paul, 200; Paul et al., 2007). Research has shown perceptual and motor deficits in interhemispheric transfer of complex sensory information and learning (Brown, Jeeves, Dietrich, and Burnison, 1999), and bimanual motor coordination (Moes, Schilmoeller, \& Schilmoeller, 2008; Mueller, Marion, Paul, \& Brown, 2009). Cognitive deficiencies include weakness in complex novel problem-solving (Brown \& Paul, 2000; Brown, Anderson, Symington \& Paul, 2012), processing of subtle prosodic and semantic aspects of language (Paul, Van Lancker-Sidtis, Schieffer, Dietrich, \& Brown, 2003), and comprehension of nonliteral language (Paul et al., 2003; Brown et al., 2005a; Brown, Symington, VanLancker, Dietrich Paul, 2005b; Rehmel, Brown \& Paul, 2016), as well as slowed cognitive processing time (Marco, Harrell, Brown, et al., 2013).

In a survey of parents of children with AgCC using the Child Behavior Checklist (Achenbach \& Rescorla, 2001), a significant percent of older children with primary AgCC were reported to have clinically significant thought problems, as well as difficulties with attention and socialization (Badaruddin, Andrews, Bolte, Schilmoeller, Schilmoeller, Paul \& Brown, 2007). These reports were similar to the reports of parents of children with autism, although less severe in children with AgCC. 
In addition, these problems were not reported by parents of children with AgCC who were 6 years of age or younger.

\section{Social Processing in AgCC}

One of the prominent impairments in primary $\mathrm{AgCC}$ that is difficult to adequately measure is psychosocial competence. Research has suggested that deficits in social ability, often described by family members, show up in impairments in domains such as theory of mind (Paul, Schieffer \& Brown, 2004), recognition of emotions (Paul, Schieffer \& Brown, 2004; Paul, Lautzenhiser, Brown, Hart Neumann, Spezio \& Adolphs, 2006; Symington, Paul, Symington, Ono, \& Brown, 2010; Bridgman, Brown, Spezio, Leonard, Adolphs, \& Paul, 2014), detecting prosodic cues in spoken language (Paul et al., 2003), comprehension of non-literal language such as metaphors (Paul et al., 2003), jokes (Brown et al., 2005a) and proverbs (Rehmel, Paul and Brown, 2016), and imagining and reasoning abstractly about complex socioemotional situations (Anderson, Brown and Paul, 2017). For example, Symington and colleagues (2010) showed that individuals with primary AgCC were worse than controls at identifying emotions in naturalistic video clips, recognizing paradoxical sarcasm, and reading critical social cues in video clips. Persons with $\mathrm{AgCC}$ have also exhibited impairments in identifying emotions in semantically neutral sentences spoken with a happy, sad, angry, or surprised tone of voice (Paul et al., 2003) and identifying emotion in faces seen for only 1 second (Bridgman et al., 2014), indicating deficits in processing social cues as they occur in realtime. In terms of expressing social and emotional content, individuals with primary AgCC have been found to use fewer words pertaining to emotionality, cognitive processes, and social processes than controls (Turk, Brown, Symington, \& Paul, 2009), to be impaired in story logic, social understanding, and common content when providing narratives for the Thematic Apperception Test (TAT; Murray, 
1942; Paul, Schieffer, \& Brown, 2004), and to be deficient in recognizing and expressing their own emotions (Pazienza, 2011; Paul, Pazienza, and Brown, submitted).

Research on social functioning in $\mathrm{AgCC}$ has generated two hypotheses. First, we have thought that AgCC might be generally characterized by deficits in rapid, complex, and novel problem-solving (Brown and Paul, 2000; Paul et al., 2007), and that social situations often involve both novelty and complexity occurring at a rate that does not allow for extensive reflection. Thus, problems in social processing are apparent to the degree that the situation is new, complex, and demanding of rapid comprehension. Another, but not mutually exclusive, hypothesis suggests that persons with $\mathrm{AgCC}$ have a difficult time imaging information and implications that are not immediately apparent and explicit in the current situation. Social situations often involve implied information from previous situations, imagined future or alternatives situations, or non-explicit references in language. It is possible that persons with $\mathrm{AgCC}$ are not adept at recognizing and processing such second-order social information. For example, Symington et al. (2010) showed that persons with AgCC had difficulty interpreting the social meanings of speech and actions (presented in the videos of the The Awareness of Social Inference Test) particularly when understanding of the current situation required them to integrate their own knowledge with the perspective of another person. This study also showed that persons with AgCC had difficult understanding sarcasm, particularly paradoxical sarcasm. These social processing deficits mirror those seen in language comprehension where individuals with AgCC have difficulty understanding the second-order meanings of language in jokes, idioms, and proverbs (Brown et al., 2005ab; Paul et al., 2003; Rehmel et al., 2016). The aim of the current study was to determine if these deficits in complex problemsolving and imagination of second-order meanings in language extend to prospective imagination and expression regarding the potential consequences of one's actions in social contexts. 


\section{The Awareness of Consequences Scale}

The Awareness of Consequences Scale (AOCS; Schwartz, 1968a) is a test demanding prospective imagination regarding the potential consequences that one's behavior might have on the welfare of others when making a decision in a social context. It is composed of six vignettes, each of which involves a main character who is caught in a social dilemma and must make an immediate decision. After each story is read aloud, participants are asked to imagine what it would be like to be in the main character's position, then verbally describe what thoughts might be going through the central character's mind and what feelings he or she might be experiencing. It is the awareness of the consequences of various behavioral options that is of importance in this test.

Symington (2003) used the AOCS to compare 13 individuals with primary AgCC and 13 ageand IQ-matched controls. As was hypothesized, participants with AgCC scored significantly lower than controls. An incidental finding of this research was that the 5-point scoring system did not account for many seemingly important characteristics of the responses of individuals with AgCC. For example, although many persons with AgCC were able to express the decisions the characters in the vignettes would make, they were not able to give reasons for these decisions. Instead, they seemed to rely on an overlearned, rote understanding of socially normative behavior, but seemed unaware of the wider implications and consequences of the actions, or of how potential behaviors might be viewed by others.

\section{Semantic Analysis of Texts}

The current study expanded on Symington's (2003) study by applying methods of linguistic analysis to more fully characterize the semantic content and features of responses to the AOCS provided by neurotypical control participants and individuals with AgCC. Responses were analyzed using two forms of semantic analysis: Latent Semantic Analysis (LSA), a tool that measures semantic 
similarity between two bodies of text; and Linguistic Word and Inquiry Count (LIWC), a syntactic and semantic word-counting tool. These scoring methods have the added value of not relying on subjective judgments of the AOCS test that are often difficult to make and in some cases not consistently applied by different raters.

LSA allows for semantic comparisons between texts. LSA applies a computational algorithm to model semantic word relationships from very large corpora of texts, resulting in a highdimensional semantic space. Within this space other samples of text (phrases, sentences, paragraphs, or larger texts) can be represented as a vector. The degree of semantic similarity between two target texts is then quantified as the cosine between their two vectors (Landauer, 2007), providing an objective and quantitative measure of semantic similarity.

LSA provides text analysis that is consistent with semantic analysis and categorizaion conducted through human cognition. For example, LSA has performed at a high school level on multiple-choice vocabulary tests LSA (Landauer, Foltz \& Laham, 1998) and has been shown to effectively measure recall performance on the narrative memory portion of the Wechsler Memory Scale (Lautenschlager, Dunn, Bonney, Flicker, \& Almeida, 2006). In comparison to subjective analyses condcted by humans, LSA performed similarly in assessing the content quality of expository essays (Landauer, Laham, \& Foltz, 2003). Similarly, LSA analysis of speech passages accurately identified individuals who had been professionally diagnosed with schizophrenia (Brita, Foltz, Weinberger, \& Goldberg, 2007).

LSA has also been used to identify the degree of specific semantic content in passages of text. This approach was used to assess the self-representations embedded in narrative responses provided by individuals who were deemed to be exemplars of compassion based on a lifetime of caring for mentally and/or physically handicapped individuals (Birath, 2010; Reimer, 2004; Reimer, Young, 
Birath, Spezio, Peterson, Van Slyke \& Brown, 2012; Young, 2010). That is, to assess the degree to which the semantics of various virtues were embedded in the self-identity narratives of caregivers, Reimer et al. (2012) compared narrative responses to target texts representing caring, justice, bravery, and religiousness, finding that these domains of semantic content increased in the self-representations of caregivers with greater years of service.

Characterization of the usage of specific categories of semantic content in AOCS responses was addressed through use of LIWC2001 (Pennebaker, Francis, \& Booth, 2001). Words are categorized and counted by grammatical dimensions (e.g., percentage of pronouns, articles), psychological constructs (e.g., affective or cognition words), dimensions related to "relativity" (e.g., time, space, motion), and personal concern categories (e.g., work, home, leisure activities).

LIWC has been used to measure psychosocial competence in individuals with neurological abnormalities. Heberlein, Adolphs, Pennebaker, and Tranel (2003) analyzed the spontaneous verbal descriptions of individuals with damage to the somatosensory cortex of the right-hemisphere to short movies depicting objects that move in socially suggestive ways. Results indicated that individuals with this form of cortical damage reacted to the stimuli with fewer words describing emotional and social processes than did controls without brain damage, despite using a similar total number of words.

LIWC was also used to examine social comprehension in $\mathrm{AgCC}$ by assessing responses to the cards of the Thematic Apprehension Task (TAT) in individuals with AgCC and neurotypical controls (Turk et al., 2009). In this task, participants viewed a black-and-white drawings depicting a social scene and were asked to tell a story about each picture. The LIWC analysis revealed atypical word usage by the AgCC group in both syntactic and semantic categories. With regard to syntax, individuals with $\mathrm{AgCC}$ used relatively more present tense verbs and first person pronouns. With 
regard to semantic content, the participants with $\mathrm{AgCC}$ did not differ from control participants on frequency of references to personal concerns (Occupation; Leisure activity; Money and Financial issues; Metaphysical issues; and Physical States and Functions) or words describing sensory perceptual processes. However, individuals with $\mathrm{AgCC}$ used fewer words than controls pertaining to emotional, cognitive, and social processes. These findings suggested that callosal agenesis results in deficiencies in imagining and inferring the mental, emotional, and social functioning of others as implied by TAT pictures, and in translating this content into a narrative that describes events beyond their present and first person perspective. In keeping with results from this study of TAT responses, the current study used LIWC to examined general features of language use and syntax, as well as six specific semantic categories: emotional processes, cognitive processes, social processes, sensory processes, and physical states.

\section{Aims and Hypotheses}

The goal of the current study was to clarify the nature of social and emotional cognition in $\mathrm{AgCC}$ with respect to imagination of the consequences of decisions. Thus responses on the AOCS from individuals with primary AgCC were analyzed using the standard subjective scoring system, and two sensitive and objective tools of text analysis: LSA and LIWC. It was hypothesized that, similar to Symington's (2003) findings, individuals with primary AgCC would score lower on the AOCS when using the 5-point scoring system developed by Schwartz (1974). We hypothesized that LSA would show that the level of linguistic similarity between participant responses and target responses was lower in the AgCC group than controls, and that LSA cosines would correlate positively with scores from the Schwartz scoring system. Finally, we hypothesized that LIWCm would show that individuals with AgCC would use fewer words pertaining to emotional, cognitive, and social processes than controls, similar to the outcome of Turk et al. (2009). 


\section{Method}

\section{Participants}

This study analyzed AOCS responses of 28 participants with primary AgCC (21 complete and 7 partial AgCC) and 32 neurotypical controls. Demographics for each group are presented in Table 1. The AgCC group did not differ from the control group on age, WAIS-III Full Scale Intelligence Quotient (FSIQ), Verbal Comprehension Index (VCI), POI (Perceptual Organization Index), education, or gender. Persons with AgCC were recruited through the ACC Network and the National Organization of Disorders of the Corpus Callosum or were self-referrals. Control participants were recruited from responders to a Craigslist.com advertisement and were included based on an initial telephone screening. Schwartz system scores for thirteen of the persons with AgCC and 13 of the controls in this study were also reported in Symington (2003). However, the raters who generated the Schwartz scores for Symington (2003) paper were not available to rate the additional responses included in this study, therefore two new raters applied the Schwartz scoring method to all responses included herein.

Inclusionary criteria for participants with $\mathrm{AgCC}$ consisted of MRI or CT scan verification of a complete or partial absence of the corpus callosum, and an FSIQ of at least 80. Exclusionary criteria for both AgCC and control participants included English as a second language, history of moderateto-severe head injury, intractable epilepsy, and drug abuse as assessed by clinical interview. Control participants were also excluded if they had a known CNS disorder or structural brain abnormality and participants with AgCC were excluded for presence of structural brain abnormality visible on MRI other than that which is typically found in $\operatorname{AgCC}$ (e.g., colpocephaly, Probst bundles, and occasional small heterotopias). Potential control participants were screened via a telephone interview and administration of the vocabulary subtest of the WAIS-III (Wechler, 1997) to determine if they were 
likely to meet the exclusionary criterion of an 80 or higher FSIQ. All participants were given a current age-appropriate Wechsler intelligence test as a part of laboratory testing.

Test results were gathered as part of a larger test battery utilized to investigate the cognitive and psychosocial consequences of AgCC. Each participant read and signed a consent form describing the research. Methods for this study were approved by Institutional Review Board of the Travis Research Institute.

\section{Measures}

The Awareness of Consequences Scale (AOCS; Schwartz, 1974) was designed to investigate the awareness of the potential consequences of one's acts on the welfare of others during decisionmaking. It is composed of six stories wherein the central character is faced with a decision. Stories are presented one at a time and participants are instructed to imagine what it would be like if they were in the central character's position. Following each story, examinees are asked to think aloud and describe what thoughts might be going through the central character's mind and what feelings he or she might be experiencing.

The vignettes of the AOCS get progressively more complex from vignette 1 to 6 . For example, in vignette 1 the dilemma focuses on competing priorities within the principle character (i.e. either get out of bed to fulfill a social obligation with friends or sleep longer instead). In contrast, vignette 4 requires the respondent to not only imagine the personal consequences of a decision, but also the potential consequences for another person whose reason for distress is undisclosed in the vignette itself. Finally, vignette 6 introduces an ethical dilemma that requires consideration of potential personal gain, as well as potential long-term impact on the well-being of many unknown individuals. Thus, the vignettes increase in complexity by introducing additional persons who may be 
impacted by the decision, and greater amounts of uncertainty regarding when and how these impacts may occur.

The Schwartz System of Scoring for the AOCS is based on a 5-point scale assessing the extent to which the actor was aware of the potential consequences of his or her behavior on the welfare of others. A response that indicates the absence of awareness of consequences for the welfare of others is given a score of 0 . A score of 1 indicates awareness of a general norm or obligation not to harm others, but without expression of more specific potential effects. A score of 2 is given when the participant wonders what it would be like to be in the position of the other person in the story without actually describing possibilities. A score of 3 is given to responses expressing clear sympathy or emotional empathy for the main character's position, without adopting this perspective. A score of 4 is given to responses that mention specific effects on others in considerable detail and long rangeconsequences for others.

Scores on the AOCS have been found to be positively correlated with peer ratings of helpfulness and considerateness in college students (Schwartz, 1968b), and with volunteer behavior in college students solicited to participate in a charitable cause (Harland, Staats, and Wilke, 2007; Schwartz, 1974). Ascription of responsibility to the self has also been positively correlated with AOCS scores (Harland, Staats, and Wilke, 2007; Schwartz, 1968b).

In the current study, LSA was used to provide an objective and quantitative overall index of semantic normality of the responses of persons with AgCC. Each participant's AOCS responses were compared with a text composed of typical responses provided by neurotypical control participants. The resultant cosine values indicated the degree to which a participant's responses were broadly semantically similar to the target paragraphs that were gleaned from the responses of controls. In this manner, LSA provided an overal index of semantic normality, but did not provide any detailed 
information about the semantic content provided by either controls or individuals with AgCC.

Latent Semantic Analysis was used in this research as a way to derive an objective measure of the normality of the overall semantic content of responses of persons with AgCC. LSA was implemented in this research using the 300-dimensional semantic space derived from the Educator's Word Frequency Guide of Touchstone Applied Science Associates [TASA], an 11-million-word representative sample of a wide variety of texts expected to be read by students from first grade through the first year of college (Zeno, Ivens, Millard, \& Duvvuri, 1995). Responsese from participants were compared to a target text that represented a common semantic core for the control group for each vignette (procedure described below). Thus, the transcribed responses of each participant ( $\mathrm{AgCC}$ and control) for each vignette were compared to the target (common core) response within the TASA high-dimensional semantic space.

LIWC2001 (Pennebaker, Francis, \& Booth, 2001) was used to compare the frequency with which participants with $\mathrm{AgCC}$ and control participants used words in specific lexical/semantic and syntactic categories. LIWC2001 matches each word in a text sample to a dictionary containing word stems (i.e. words stripped of prefixes and suffixes) which have been categorized according to syntactic and/or semantic features. An example of a word stem is "hungr*" which allows for any target word that matches this stem to be counted as an eating word (e.g., hungry, hungrier, hungriest). For each category, frequency of use is calcualted relative to the total number of words in that text sample. Not all the words are tagged as dictionary words. Categories addressed in this research involved general characteristics of texts (e.g., total words); syntactic features of words (e.g., part of speech); and 6 semantic categories of theoretical relevance to $\mathrm{AgCC}$ (emotional processes, cognitive processes, social processes, sensory processes, and physical states).

\section{Procedures}


The participant was given a printed copy of the AOCS vignettes. The administrator read the instructions verbatim, and then read aloud each story as the participant followed along. After each story, the participant was asked to describe what thoughts might be going through the central character's mind and what feelings he or she might be experiencing. After their response, the participant was asked, “Anything else?" before proceeding to the next story. Responses were written down verbatim, then transcribed for scoring and semantic analyses.

Schwartz System Scoring: De-identified transcripts was scored by two trained raters (neither of whom provided ratings for the previous report, Symington 2003). The raters obtained an inter-rater reliability of $\rho_{I}=.72$. Discrepancies in ratings of 2 points or more were discussed by the raters and replaced with a consensus score. Otherwise, the average of the scores from the two raters for each item for each vignette was used as the participant's Schwartz System score.

Latent Semantic Analysis: Participants' transcribed responses were “cleaned” so as to be appropriate for LSA - that is, all contractions were expanded, commas were deleted, and all ending punctuations were replaced with periods. Target paragraphs for each vignette were developed using a matrix comparison that measured the degree of similarity of each control response to the responses of all other controls. That is, for each vignette, every control response that rendered an average cosine of .51 or greater when compared to responses of all other controls was included in the target paragraph, resulting in a different number of contributions to the target paragraph of each vignette (vignette $1, n$ $=23$; vignette $2, n=13$; vignette $3, n=21$; vignette $4, n=10$; vignette $5, n=5$; vignette $6, n=7$ ). Thus, target paragraphs represented the most common semantic content in the responses of the control group to each vignette. The scenarios presented in vignettes 4, 5 and 6 are situationally more complex and therefore elicit a greater variety of responses among controls. Thus, the inter-participant cosigns for controls tended to fall below .51 more frequently for these vignettes. 
For each vignette, the responses of each AgCC participant were compared to the target paragraph using LSA. Similarly, the responses of each control participant that were not included in the target paragraph were compared to the target paragraph. Responses from the controls that were included in the target paragraph were compared to the target paragraph that was remaining after removing that participant's own response from the ensemble target paragraphs. Thus, each AOCS response rendered a cosine that represented the degree of semantic similarity between that response and the target text within the high-dimensional TASA semantic space.

Linguistic Inquiry and Word Count: Participants' transcripts “cleaned" for LSA were assessed with LIWC, producing subject-wise word-category frequencies for each separate vignette and for all vignettes combined. For this study, LIWC results for 3 types of categories were analyzed: (1) General characteristics of the protocols, including the total number of words, the percentage of dictionary words, and the percentage of unique words; (2) Syntactic categories denoting syntactic complexity, including percentage of words with six letters or more, negations, assents, verbs (past, present and future), pronouns as a whole, pronouns divided into first, second and third person, articles, prepositions, and numbers; and (3) Semantic categories, including emotional processes, cognitive processes, social processes, sensory processes, and physical states. Word frequencies in each category were expresses and analyzed as percentages of the total words in the response.

\section{Results}

\section{Awareness of Consequences Scores}

Due to skewness in the Schwartz scores, a Mann-Whitney U was used to test group differences on total score for all vignettes and on each vignette. Total scores in the AgCC group were significantly lower than the control group, with a medium effect size (Mann-Whitney $\mathrm{U}, \mathrm{d}_{\text {Cohen }}=.53$; see Table 2). In comparisons of each vignette, individuals with $\mathrm{AgCC}$ had significantly lower scores than controls on 
vignettes 5 and 6 , also with medium effect sizes $\left(\mathrm{d}_{\text {Cohen }}=.61\right.$ and .50$)$. There was also a weak trend toward lower scores on vignette 4 in the $\mathrm{AgCC}$ group $\left(\mathrm{d}_{\mathrm{Cohen}}=.34\right)$, but their scores were not different from controls for vignettes 1,2 , and 3 .

\section{Latent Semantic Analysis}

Group comparison of LSA cosines (comparing individual responses to target texts) for the 6 vignettes was conducted using multivariate analysis of variance (MANOVA). Over all vignettes, cosines were lower in the AgCC group with a large effect size, but not quite reaching significance, $\eta_{\mathrm{p}}{ }^{2}=.20, F(6,53)=2.27, p=.051$. Examined separately, cosines for vignettes 4 and 6 were significantly lower in the AgCC group than the control group, with medium effect sizes $\left(\eta_{\mathrm{p}}{ }^{2}=.09\right.$ and $\eta_{\mathrm{p}}{ }^{2}=.08$, respectively; see Table 3$)$.

LSA cosines were compared to Schwartz scores across vignettes and groups. A moderate but significant correlation was found (Pearson's $r=.28, p=.032$ ), confirming the hypothesis that the level of semantic similarity between individual participant responses and target responses correlates positively with the subjective Schwartz system ratings.

\section{Linguistic Inquiry and Word Count}

In analysis of responses to all vignettes, participants with $\mathrm{AgCC}$ did not differ significantly from controls with respect to any of the general text characteristics of total word count, relative frequency of dictionary words, the proportion of words over 6 letters in length, or relative frequency of unique words. The group with AgCC also did not differ significantly from controls with respect to most syntactic categories, including negation or assent words, verbs in past, present or future tense, pronouns, or proportion of articles or prepositions. However, participants with AgCC were significantly more likely to use words denoting numbers, $\eta_{\mathrm{p}}{ }^{2}=.29, F(1,58)=8.60, p=.005$. 
In analyzing semantic content categories, we focused on six categories of theoretical interest in $\mathrm{AgCC}$ : emotional processes, cognitive processes, social processes, sensory processes, and physical states. Compared to controls, responses from participants with AgCC contained a significantly smaller proportion of words from the emotional processes category. Although not significantly different, the responses of participants with $\mathrm{AgCC}$ suggested lesser usage of cognitive process words and a greater use of words referring to physical states (see Table 4).

Table 5 shows the results of post-hoc group comparisons for each sub-category of emotional processes, as well as exploratory comparisons of cognitive process and physical state sub-categories. Within the emotion category, responses of participants with AgCC were less likely than control responses to contain negative emotion words (with a trend toward reduced use of words related to sadness). Within the cognitive process category, words referencing insight were used significantly less often by persons with $\mathrm{AgCC}$, but the groups did not differ in use of any other cognitive process or physical state sub-categories.

Finally, for the vignettes that garnered group differences on Schwartz scoring (vignette 5) or LSA cosines (4 and 6), we compared LIWC semantic categories between groups. Multivariate ANOVA with the 5 semantic categories revealed a significant group difference for vignette $4, \mathrm{~h}_{\mathrm{p}}{ }^{2}=$ $.218, F(5,54)=3.01, p=.018$, but not vignettes 5 or $6, \mathrm{~h}_{\mathrm{p}}{ }^{2}=.114, F(5,54)=2.384, p=.245$ and $_{\mathrm{p}}{ }^{2}$ $=.010, F(5,54)=.105, p=.991$. On vignette 4, the AgCC group was less likely than control group to use words related to cognitive processes, $\mathrm{h}_{\mathrm{p}}{ }^{2}=.098, F(1,176.36)=6.311, p=.015(\mathrm{AgCC}$ mean $=$ 10.075, $\mathrm{sd}=55.28$; Control mean $=13.512, \mathrm{sd}=5.29)$, but did not differ for any other semantic category. Although there was not an overall effect for vignette 5, the AgCC group was less likely than the control group to use emotion words for this vignette, $\mathrm{h}_{\mathrm{p}}{ }^{2}=.087, F(1,35.865)=5.499, p=$ .022 . 


\section{Discussion}

The aim of the current study was to determine if deficits in complex problem-solving and imagination of second-order meanings in language extend to prospective imagination and expression regarding the potential consequences of one's actions in social contexts. Thus, this study investigated deficits in social awareness and problem solving in individuals with AgCC using linguistic analyses of responses to the Awareness of Consequences Scale. The AOCS assesses the capacity to anticipate the consequences of one's actions, including feelings regarding oneself and others associated with potential actions. As predicted, individuals with AgCC scored significantly lower when using the typical scoring of the AOCS, had divergent semantic content in their responses compared to the common responses of controls, and their responses contained a lower proportion of words referring to emotions. These results are consistent with previous research indicating that individuals with AgCC have difficulties with understanding and imagining the emotions and cognitions of others in complex social contexts (Turk et al., 2009).

\section{Awareness of Consequences Scores}

Using the subjective judgments of the Schwartz scoring system, this study replicated (with a much larger group) the findings of Symington (2003) that individuals with AgCC performed more poorly than controls on the AOCS. The problem with this scoring system is that the subjective ratings are, in some cases, difficult to apply and may cause raters to disagree. Nevertheless, the results of this scoring system in this study are consistent with the previous finding that the stories told by individuals with $\mathrm{AgCC}$ when prompted by the pictures on TAT cards had a tendency to be deficient in descriptions of social implications, speculation about past and future experiences of the people pictured, and inferences of the emotions of persons in their narratives (Paul et al., 2004; Turk et al., 2009). 


\section{Latent Semantic Analysis}

The hypothesis that individuals with AgCC would tend to produce semantically atypical responses to the AOCS vignettes was generally supported. For responses combined over all vignettes, LSA cosines (derived from comparison to the target text) were lower in the AgCC group than the control group. The positive correlation between Schwartz scores and the combined vignette LSA cosines suggests that the less-normative responses of individuals with AgCC likely involved less semantic content related to how one's decisions can affect others, and what one might be feeling with respect to potential outcomes (i.e., the criterion for scoring judgments in the Schwartz system).

Group comparison of LSA cosines from each vignette revealed a noteworthy pattern. The AgCC group had significantly lower cosines than controls on vignettes 4 and 6 , as well as a small but not significant difference on vignette 5 , but they clearly did not differ from controls on the first three vignettes. This pattern provides insight into the nature of processing deficits that impact social problem-solving in individuals with AgCC since the vignettes in the AOCS get progressively more complex and difficult to imagine. With this increasing complexity, the responses of individuals with AgCC get more discrepant from the common responses of controls, indicating that their problemsolving limitations are most apparent when they must consider multiple perspectives and make more substantial inferences about uncertain outcomes.

The fact that these LSA cosines correlated moderately but significantly with the outcome of the Schwartz Scoring System suggests that LSA has the potential to provide an alternative to this subjective scoring system. For the purpose of the current study a target comparison response was assembled from the sub-group of controls whose responses were most alike (not unlike the central tendency of scores in a normative population). However, given the promising LSA results of this research, it would be worthwhile exploring various kinds of target texts that might resemble even 
more strongly the subjective scoring outcome, but nevertheless provide an objective scoring procedure.

\section{Linguistic Inquiry and Word Count}

LSA confirmed that AOCS responses provided by individuals with AgCC are semantically different than responses given by controls, but offered only limited insight regarding the specific factors that contribute to this difference. To probe the nature of AOCS responses more precisely with respect to fundamental language use and domains of semantic content, we analyzed the same collection of resposnes using LIWC (2001). With respect to general characteristics and syntactic properties of their responses, persons with $\mathrm{AgCC}$ seemed to provide responses with similar quantity and general sentence quality as did controls. However, the responses of individuals with AgCC differed from the control group in usage of words that describe emotional responses and aspects of the cognitive processes of the characters in the vignettes.

The AgCC group was significantly less likely to use words reflecting emotions (specifically negative emotions), and fewer words reflecting cognitive processes. This outcome may reflect difficulty imagining the emotional experiences and cognitive processes that are likely to be occurring in the mind of someone else as he or she considers the consequences of potential actions - or, stated more succinctly, impaired theory of mind. In a previous study using The Awareness of Social Inference Test, we also found that individuals with $\mathrm{AgCC}$ were deficient in the recognition of emotions (specifically negative emotions) and had difficulty integrating multiple sources of information when interpreting social interactions (Symington et al., 2010), but were able to accurately interpret simple social interactions. Taken together, this previous study and the current findings support the hypothesis that AgCC results in limited capacity for complex theory of mind and problem solving in a social context. 
Reduced usage of words reflecting emotional processes is also consistent with recent work suggesting alexithymia in individuals with AgCC. Alexithymia is defined as deficient emotional expressiveness, difficulty identifying and expressing feelings, tendency towards somatic complaints, concrete and externally oriented thinking style, and lack of imaginative thinking. Individuals with AgCC have been shown to score higher than neurotypical controls on the Toronto Alexithymia Scale and to show a greater quantity of somatic complaints on the Symptom Checklist-90-R (Pazienza, 2011; Paul, Pazienza, and Brown, submitted). Also consistent with alexithymia was the tendency (not significant) for individuals with $\mathrm{AgCC}$ in the current study to use more words than controls pertaining to physical states and bodily functions. This is interesting because an important aspect of the interhemispheric transfer deficit model of alexithymia posits that the processing of emotionally laden material in the right hemisphere becomes cut off from the verbally expressive left hemisphere and instead gets expressed via bodily symptoms (Tabibnia \& Zaidel, 2005). This theory is consistent with our finding that individuals with $\mathrm{AgCC}$ were less likely than controls to use words referencing emotions, although on the AOCS only marginally more likely to use words referring to physical states.

The hypothesis that individuals with AgCC would use fewer social words than controls was not supported. The social processes category of the LIWC included words that pertained to communication (e.g., "talk," "share," "converse"), references to people (e.g., second or third person pronouns), friends (e.g., "pal," "buddy”), family (e.g., "mom," "brother”), and humans (e.g., "boy," "woman”). Unlike the Turk et al. (2009) study which showed that individuals with AgCC used fewer words expressing social processes when telling stories based on TAT pictures, the current study found no differences between AgCC participants and controls in their use of social words. The discrepancy between these results may simply be a product of task differences: the TAT involves pictures of persons apparently interacting, and thus would normally elicit stories involving social interactions. In contrast, the vignettes and the 
task requirements for the AOCS (i.e., to "think out loud what a character might be thinking or feeling") are not as explicitly social, but are more related to individual behaviors and their likely consequences. Thus, the AOCS is probably less effective than the TAT for testing participants' understanding of, or focus on, social interactions. This speculation is supported by the fact that the control group's frequency of using social process words was $20.7 \%$ on the TAT (Turk et al., 2009), but for controls in the present study, the frequency of social process words was only $12.6 \%$. Thus, whether or not deficiency in inferences of social processes is characteristic of $\mathrm{AgCC}$ is not well tested by the AOCS.

\section{Conclusions}

The results of this study continue to support both of the not-mutually-exclusive explanations of deficits in social functioning in individuals with AgCC. The fact that differences from neurotypical controls emerged when responding to the more complex vignettes (4-6) continues to support a focus on deficiencies in complex novel problem-solving. However, given the nature of the AOCS, these data also demonstrate that persons with AgCC have difficulty imaging and explaining the likely consequences of behavioral choices. These explanations interact in that the consequences of more straightforward choices are easier for them to imagine and explain. An additional element is that their explanations are particularly deficient in representations of the emotions that might be involved.

This research was somewhat limited by the nature of the AOCS. The responses elicited by the vignettes are typically not long (in the neighborhood of 50 words), resulting in a smaller quantity of text for these forms of semantic analyses. Also, as indicated above, the vignettes do not require imagination of richer forms of social interactions. Given that $\mathrm{AgCC}$ is somewhat rare, it is difficult to identify sufficient cases for amassing large research groups. Nevertheless, the size of the current 
AgCC participant group provided sufficient statistical power to reveal important weakness in their awareness of the consequences of different forms of behavioral decisions. 


\section{References}

Achenbach, T. M., \& Rescorla, L. A. (2001). Manual for the ASEBA School-Age Forms \& Profiles. Burlington, VT: University of Vermont, Research Center for Children, Youth, \& Families. Anderson, L. B., Paul, L. K., \& Brown, W. S. (2017). Emotional intelligence in agenesis of the corpus callosum. Archives of Clinical Neuropsychology, 32, 267-279. doi:10.1093/arclin/acx001

Badaruddin, D. H., Andrews, G. L., Bolte, S., Schilmoeller, K. J., Schilmoeller, G., Paul, L. K., \& Brown, W. S. (2007). Social and behavioral problems of children with agenesis of the corpus callosum. Child Psychiatry and Human Development, 38, 287-302. doi:10.1007/s10578-0070065-6

Birath, J. B. (2010). Virtue with respect to self over time: A comparison of long-term and short-term L'Arche caregivers (Unpublished master's thesis). Fuller Graduate School of Psychology, Pasadena, CA.

Bridgman, M. W., Brown, W. S., Spezio, M. L., Leonard, M. K., Adolphs, R., \& Paul, L. K. (2014). Facial emotion recognition in agenesis of the corpus callosum. Journal of Neurodevelopmental Disorders, 6, 32. doi:10.1186/1866-1955-6-32

Brown, W. S., Anderson, L. B., Symington, M. F., \& Paul, L. K. (2012). Decision-making in individuals with agenesis of the corpus callosum: Expectancy-valence in the Iowa Gambling Task. Archives of Clinical Neuropsychology: The Official Journal of The National Academy of Neuropsychologists, 27, 532-544. doi:10.1093/arclin/acs052

Brown, W. S., Jeeves, M. A., Dietrich, R., \& Burnison, D. S. (1999). Bilateral field advantage and evoked potential interhemispheric transmission in commissurotomy and callosal agenesis. Neuropsychologia, 37, 1154-1180. doi:10.1016/S0028-3932(99)00011-1 
Brown, W. S., \& Paul, L. K. (2000). Cognitive and psychological deficits in agenesis of the corpus callosum with normal intelligence. Cognitive Neuropsychiatry, 5, 135- 157. doi:10.1080/135468000395781

Brown, W. S., Paul, L. K., Symington, M., \& Dietrich, R. (2005a). Comprehension of humor in agenesis of the corpus callosum. Neuropsychologia, 43, 906-916. doi:10.1016/j.neuropsychologia.2004.09.008

Brown, W. S., Symington, M., VanLancker, D., Dietrich, R. and Paul, L. K. (2005b) Paralinguistic processing in children with Callosal Agenesis: Emergence of neurolinguistic deficits. Brain and Language, 93, 135-139. doi:10.1016/j.bandl.2004.09.003

Chiarello, C. (1980). A house divided? Cognitive functioning with callosal agenesis. Brain and Language, 11, 128-158. doi:10.1016/0093-934X(80)90116-9

Derogatis, L. R. (1994). SCL-R: Symptom Checklist-90-R: Administration, scoring, and procedures manual (3rd ed.). Minneapolis, MN: National Computer Systems.

Elvevag, B., Foltz, P. W., Weinberger, D. R., \& Goldberg, T. E. (2007). Quantifying incoherence in speech: An automated methodology and novel application to schizophrenia. Schizophrenia Research, 93, 30-316. doi:10.1016/j.schres.2007.03.001

Folsom, R. (2009). Awareness of consequences in individuals with agenesis of the corpus callosum (Unpublished master's thesis). Fuller Graduate School of Psychology, Pasadena, CA.

Glass, H. C., Shaw, G. M., Ma, C., \& Sherr, E. H. (2008). Agenesis of the corpus callosum in California 1983-2003: A population-based study. American Journal of Medical Genetics, 146, 2495-2500. doi:10.1002/ajmg.a.32418

Griffiths, T. L., Steyvers, M., \& Tenenbaum, J. B. (2007). Topics in semantic representation. Psychological Review, 114, 211-244. doi:10.1037/0033-295X.114.2.211 
Guillem, P., Fabre, B., Cans, C., Robert-Gnansia, E., \& Jouk, P. S. (2003). Trends in elective terminations of pregnancy between 1989 and 2000 in a French county (the Isere). Prenatal Diagnosis, 23, 877-833. doi:10.1002/pd.711

Heberlein, A. S., Adolphs, R., Pennebaker, J. W. \& Tranel, D. (2003). Effects of damage to righthemisphere brain structures on spontaneous emotional and social judgments. Political Psychology, 24, 705-726. doi:10.1046/j.1467-9221.2003.00348.x

Harland, P., Staats, H., \& Wilke, H. A. M. (2007). Situational and personality factors as direct or personal norm mediated predictors of pro-environmental behavior: Questions derived from norm-activation theory. Basic and Applied Social Psychology, 29, 323-334. doi:10.1080/01973530701665058.

Landauer, T. K. (2007). LSA as a theory of meaning. In T. Landauer, D. McNamara, S. Dennis, \& W. Kintsch (Eds.), Handbook of latent semantic analysis (pp. 3-34). Mahwah, NJ: Erlbaum.

Landauer, T. K., Foltz, P. W., \& Laham, D. (1998). An introduction to latent semantic analysis. Discourse Processes, 25, 259-284. doi:10.1080/01638539809545028

Landauer, T. K., Laham, D., \& Foltz, P. W., (2003). Automatic essay assessment. Assessment in Education, 10, 295-308. doi:10.1080/0969594032000148154

Lautenschlager, N. T., Dunn, J. C., Bonney, K., Flicker, L. \& Almeida, O. P. (2006). Latent semantic analysis: An improved method to measure cognitive performance in subjects of non-Englishspeaking-background. Journal of Clinical and Experimental Neuropsychology, 28, 13811387. doi:10.1080/13803390 500409617

Marco, E. J., Harrell, K. M., Brown, W. S., Hill, S. S., Jeremy, R. J., Kramer, J. H., .. Paul, L. K. (2012). Processing speed delays contribute to executive function deficits in individuals with agenesis of the corpus callosum. Journal of the International Neuropsychological Society, 18, 
521-529. doi:10.1017/S1355617712000045

Moes, P., Schilmoeller, K., \& Schilmoeller, G. (2008). Physical, motor, sensory and developmental features associated with agenesis of the corpus callosum. Child: Care, Health and Development, 35, 656-672. doi:10.1111/j.1365-2214.2009.00942.x

Oakes Mueller, K. L., Marion, S. D., Paul, L. K., \& Brown, W. S. (2009). Bimanual motor coordination in agenesis of the corpus callosum. Behavioral Neuroscience, 123, 1000-1011. doi: $10.1037 / \mathrm{a} 0016868$

Murray, H. A. (1942) Thematic Apperception Test Manual. Cambridge, MA: Harvard University Press.

Paul, L. K., Brown, W. S., Adolphs, R., Tyszka, J. M., Richards, L. J., Mukherjee, P., \& Sherr, E. H. (2007). Agenesis of the corpus callosum: Genetic, developmental and functional aspects of connectivity. Nature Reviews Neuroscience, 8, 287-299. doi:10.1038/ nrn2107

Paul, L. K., Lautzenhiser, A., Brown, W. S., Hart, A., Neumann, D., Spezio, M., \& Adolphs, R. (2006). Emotional arousal in agenesis of the corpus callosum. International Journal of Psychophysiology, 61, 47-56. doi:10.1016/j.ijpsycho. 2005.10.017

Paul, L. K., Pazienza, S. \& Brown, W. S. (2018). Alexithymia and somatization in agenesis of the corpus callosum. Manuscript submitted for publication.

Paul, L. K., Schieffer, B., \& Brown, W. S. (2004). Social processing deficits in agenesis of the corpus callosum: Narratives from the Thematic Apperception Test. Archives of Clinical Neuropsychology, 19, 215-225. doi:10.1016/S0887-6177(03)00024-6

Paul, L. K., Van Lancker-Sidtis, D., Schieffer, B., Dietrich, R., \& Brown, W. S. (2003). Communicative deficits in agenesis of the corpus callosum: Nonliteral language and affective prosody. Brain and Language, 85, 313-324. doi:10.1016/S0093-934X(03) 00062-2 
Pazienza, S. (2011). Emotional expressiveness and somatization in agenesis of the corpus callosum (Unpublished master's thesis). Fuller Graduate School of Psychology, Pasadena, CA.

Pennebaker, J. W., Francis, M. E., Booth, R. J. (2001). LIWC 2001 manual. Mahwah, NJ: Erlbaum.

Rauch, R. A., \& Jinkins, J. R. (1994). Magnetic resonance imaging of corpus callosum dysgenesis. In M. Lassonde \& M. A. Jeeves (Eds.), Callosal agenesis: A natural split brain? (pp. 83-95). New York: Plenum Press.

Rehmel, J. L., Brown, W. S., \& Paul, L. K. (2016). Proverb comprehension in individuals with agenesis of the corpus callosum. Brain and Language, 160, 21-29. doi:10.1016/j.band1.2016.07.001

Reimer, K. (2004). Natural character: Psychological realism for the downwardly mobile. Theology and Science, 2, 35-54. doi:10.1080/1474670042000196630

Reimer, K. S., Young, C., Birath, B., Spezio, M. L., Peterson, G., Van Slyke, J., \& Brown, W. S. (2012). Maturity is explicit: Self-importance of traits in humanitarian moral identity. The Journal of Positive Psychology, 7, 36-44. doi:10.1080/174397 60.2011.626789

Sauerwein, H. C., Nolin, P. \& Lassonde, M. (1994). Cognitive functioning in callosal agenesis. In M. Lassonde \& M. A. Jeeves (Eds.). Callosal Agenesis: A natural split brain? (pp. 221-233). New York, NY: Plenum Press.

Schwartz, S. H. (1968a). Awareness of consequences and the influence of moral norms on interpersonal behavior. Sociometry, 31, 355-369. Retrieved from http://www.jstor.org/stable/i329420

Schwartz, S. H. (1968b). Words, deeds and the perception of consequences and responsibility in action situations. Journal of Personality and Social Psychology, 10, 232-242. doi:10.1037/h0026569 
Schwartz, S. H. (1974). Awareness of interpersonal consequences, responsibility denial, and volunteering. Journal of Personality and Social Psychology, 30, 57-63. doi:10.1037/h0036644

Shevell, M. I. (2002). Clinical and diagnostic profile of agenesis of the corpus callosum. Journal of Child Neurology, 17, 895-899. doi:10.1177/08830738020170122601

Symington, M. F. (2003). Social reasoning and decision making in individuals with agenesis of the corpus callosum (Doctoral dissertation). Retrieved from ProQuest Dissertations \& Theses Global. (UMI No. 3124717)

Symington, S. H., Paul, L. K., Symington, M. F., Ono, M., \& Brown, W. S. (2010). Social cognition in individuals with agenesis of the corpus callosum. Social Neuroscience, 5, 296-308. doi:2048/10.1080/17470910903462419

Tabibnia, G., \& Zaidel, E. (2005). Alexithymia, interhemispheric transfer, and right hemispheric specialization: A critical review. Psychotherapy and Psychosomatics, 74, 81-92. doi:10.1159/000083166

Turk, A. A., Brown, W. S., Symington, M., \& Paul, L. K. (2009). Social narratives in agenesis of the corpus callosum: Linguistic analysis of the thematic apperception test. Neuropsychologia, 48 , 43-50. doi:10.1016/j.neuropsychologia.2009.08.009

Wang, L. W., Huang, C. C., \& Yeh, T. F. (2004). Major brain lesions detected on sonographic screening of apparently normal term neonates. Neuroradiology, 46, 368-373. doi:10.1007/s00234-003-1160-4

Wechsler, D. (1997). WAIS-III: Wechsler Adult Intelligence Scale ( $3^{\text {rd }}$ ed.). San Antonio, TX: The Psychological Corporation.

Wechsler, D. (1999). Wechsler Abbreviated Scale of Intelligence (WASI). San Antonio, TX: The Psychological Corporation. 
Young, C. (2010). Social schemas of moral exemplars: Latent semantic analysis of interviews with L'Arche caregivers scale (Unpublished master's thesis). Fuller Graduate School of Psychology, Pasadena, CA.

Young, C. (2012). Social cognition in agenesis of the corpus callosum: Latent semantic analysis of the Awareness of Consequences Scale (Unpublished doctoral dissertation). Fuller Graduate School of Psychology, Pasadena, CA.

Zeno, S. M., Ivens, S. H., Millard, R. T., \& Duvvuri, R. (1995). The educator's word frequency guide. Brewster, NY: Touchstone Applied Science, Inc. 


\section{Table 1}

Summary of Demographic Descriptive Statistics

\begin{tabular}{|c|c|c|c|c|c|c|}
\hline & \multicolumn{3}{|c|}{$\begin{array}{c}A g C C \\
(n=28)\end{array}$} & \multicolumn{3}{|c|}{$\begin{array}{l}\text { Control } \\
(n=32)\end{array}$} \\
\hline & $M$ & $S D$ & Range & $M$ & $S D$ & Range \\
\hline Age & 29.89 & 10.36 & $17-55$ & 26.59 & 8.69 & $17-51$ \\
\hline FSIQ & 97.57 & 13.37 & $80-129$ & 101.09 & 7.57 & $84-116$ \\
\hline $\mathrm{VCI}^{\wedge}$ & 98.27 & 17.70 & $67-131$ & 102.13 & 9.49 & $87-126$ \\
\hline $\mathrm{POI}^{\wedge}$ & 102.35 & 13.75 & $76-133$ & 101.23 & 10.46 & $76-118$ \\
\hline Education & 12.96 & 2.06 & $10-18$ & 13.34 & 1.77 & $9-16$ \\
\hline Gender ( $\%$ male $)$ & $64 \%$ & & & $72 \%$ & & \\
\hline
\end{tabular}


Table 2

Schwartz Scores: Comparison of AgCC and Control Groups

\begin{tabular}{|c|c|c|c|c|c|c|c|c|c|}
\hline \multirow[b]{2}{*}{ Vignette } & \multicolumn{3}{|c|}{$\begin{array}{c}A g C C \\
(n=28)\end{array}$} & \multicolumn{3}{|c|}{$\begin{array}{l}\text { Controls } \\
(n=32)\end{array}$} & \multirow[b]{2}{*}{$U$} & \multirow[b]{2}{*}{$d_{\text {Cohen }}$} & \multirow[b]{2}{*}{$p$} \\
\hline & $M$ & $S D$ & $95 \% C I$ & $M$ & $S D$ & $95 \% C I$ & & & \\
\hline 1 & 0.59 & 0.73 & $0.32-0.86$ & 0.48 & 0.57 & $0.28-0.68$ & 436.00 & .05 & .849 \\
\hline 2 & 0.87 & 0.59 & $0.65-1.09$ & 1.03 & 0.88 & $0.73-1.33$ & 474.50 & .10 & .676 \\
\hline 3 & 0.89 & 1.04 & $0.50-1.28$ & 0.69 & 0.59 & $0.49-0.89$ & 429.50 & .07 & .777 \\
\hline 4 & 0.61 & 0.75 & $0.33-0.89$ & 1.00 & 1.05 & $0.64-1.36$ & 536.50 & .34 & .174 \\
\hline 5 & 0.20 & 0.53 & $0.00-0.40$ & 0.49 & 0.57 & $0.29-0.69$ & 600.50 & .61 & .009 \\
\hline 6 & 0.38 & 0.60 & $0.16-0.60$ & 0.72 & 0.74 & $0.46-0.98$ & 575.00 & .50 & .043 \\
\hline Total & 3.53 & 2.36 & $2.65-4.40$ & 4.39 & 1.83 & $3.76-5.02$ & 582.00 & .53 & .046 \\
\hline
\end{tabular}


Table 3

LSA Cosines Relative to Target Text: Group Comparison of Cosines for Each Vignettes

\begin{tabular}{|c|c|c|c|c|c|c|c|c|c|}
\hline \multirow[b]{2}{*}{ Vignette } & \multicolumn{3}{|c|}{$\begin{array}{c}A g C C \\
(n=28)\end{array}$} & \multicolumn{3}{|c|}{$\begin{array}{l}\text { Controls } \\
(n=32)\end{array}$} & \multirow[b]{2}{*}{$F$} & \multirow[b]{2}{*}{$\eta_{\mathrm{p}}^{2}$} & \multirow[b]{2}{*}{$p$} \\
\hline & $M$ & $S D$ & $95 \% C I$ & $M$ & $S D$ & $95 \% C I$ & & & \\
\hline 1 & 0.68 & 0.07 & $0.64-0.71$ & 0.68 & 0.07 & $0.66-0.70$ & 0.01 & .00 & .940 \\
\hline 2 & 0.65 & 0.10 & $0.61-0.69$ & 0.66 & 0.07 & $0.64-0.68$ & 0.02 & .00 & .880 \\
\hline 3 & 0.65 & 0.07 & $0.62-0.68$ & 0.65 & 0.08 & $0.62-0.68$ & 0.04 & .00 & .849 \\
\hline 4 & 0.55 & 0.15 & $0.49-0.61$ & 0.63 & 0.09 & $0.60-0.66$ & 5.41 & .09 & .024 \\
\hline 5 & 0.56 & 0.13 & $0.51-0.61$ & 0.60 & 0.09 & $0.57-0.63$ & 1.42 & .02 & .238 \\
\hline 6 & 0.57 & 0.13 & $0.52-0.62$ & 0.63 & 0.07 & $0.61-0.65$ & 5.35 & .08 & .024 \\
\hline
\end{tabular}


Table 4

Select LIWC Semantic Categories: Group Comparisons of Word Frequencies for Combined Vignettes

\begin{tabular}{|c|c|c|c|c|c|c|c|c|c|}
\hline \multirow[b]{2}{*}{$\begin{array}{l}\text { Semantic } \\
\text { categories }\end{array}$} & \multicolumn{3}{|c|}{$\begin{array}{c}\mathrm{AgCC} \\
(n=28)\end{array}$} & \multicolumn{3}{|c|}{$\begin{array}{l}\text { Control } \\
(n=32)\end{array}$} & \multirow[b]{2}{*}{$F$} & \multirow[b]{2}{*}{$\eta_{\mathrm{p}}^{2}$} & \multirow[b]{2}{*}{$p$} \\
\hline & $M$ & $S D$ & $95 \% C I$ & $M$ & $S D$ & $95 \% C I$ & & & \\
\hline $\begin{array}{l}\text { Emotional } \\
\text { processes }\end{array}$ & 3.11 & 1.17 & $2.68-3.54$ & 3.94 & 1.25 & $3.51-4.37$ & 7.08 & .11 & .010 \\
\hline $\begin{array}{l}\text { Cognitive } \\
\text { processes }\end{array}$ & 12.52 & 2.41 & $11.63-13.41$ & 13.65 & 3.19 & $12.55-14.75$ & 2.34 & .04 & .132 \\
\hline Social processes & 13.52 & 4.55 & $11.82-15.22$ & 12.57 & 4.17 & $11.17-13.97$ & 0.70 & .01 & .406 \\
\hline Sensory processes & 1.99 & 0.93 & $1.65-2.33$ & 2.05 & 1.02 & $1.70-2.40$ & 0.05 & .00 & .819 \\
\hline Physical states & 0.74 & 0.58 & $0.53-0.95$ & 0.57 & 0.38 & $0.44-0.70$ & 1.74 & .03 & .192 \\
\hline
\end{tabular}


Table 5

Group Comparison of Word Frequency within Subcategories of Emotional and Cognitive Processes Across All Vignettes

\begin{tabular}{|c|c|c|c|c|c|c|c|c|c|}
\hline \multirow[b]{2}{*}{ Semantic categories } & \multicolumn{3}{|c|}{$\begin{array}{c}\mathrm{AgCC} \\
(n=28)\end{array}$} & \multicolumn{3}{|c|}{$\begin{array}{l}\text { Control } \\
(n=32)\end{array}$} & \multirow[b]{2}{*}{$F$} & \multirow[b]{2}{*}{$\eta_{\mathrm{p}}^{2}$} & \multirow[b]{2}{*}{$p$} \\
\hline & $M$ & $S D$ & $95 \% C I$ & $M$ & $S D$ & $95 \% C I$ & & & \\
\hline \multicolumn{10}{|l|}{ Emotional Processes } \\
\hline Positive & 1.65 & 1.08 & $1.25-2.05$ & 1.98 & 1.05 & $1.62-2.34$ & 1.40 & .02 & .242 \\
\hline Negative & 1.46 & 0.80 & $1.16-1.76$ & 1.95 & 0.99 & $1.61-2.29$ & 4.41 & .07 & .040 \\
\hline Anxiety & 0.24 & 0.36 & $0.11-0.37$ & 0.34 & 0.51 & $0.16-0.52$ & 0.84 & .01 & .364 \\
\hline Anger & 0.60 & 0.60 & $0.38-0.82$ & 0.59 & 0.40 & $0.45-0.73$ & 0.00 & .00 & .961 \\
\hline Sadness & 0.20 & 0.29 & $0.09-0.31$ & 0.35 & 0.39 & $0.21-0.49$ & 2.89 & .05 & .094 \\
\hline \multicolumn{10}{|l|}{ Cognitive Processes } \\
\hline Causation & 1.34 & 0.94 & $0.99-1.69$ & 1.37 & 0.72 & $1.12-1.62$ & 0.03 & .00 & .868 \\
\hline Insight & 2.57 & 1.34 & $2.07-3.07$ & 3.29 & 1.27 & $2.85-3.73$ & 4.47 & .07 & .039 \\
\hline Discrepancy & 6.64 & 1.96 & $5.91-7.37$ & 7.35 & 2.27 & $6.56-8.14$ & 1.68 & .03 & .201 \\
\hline Inhibition & 0.67 & 0.45 & $0.50-0.84$ & 0.52 & 0.42 & $0.37-0.67$ & 1.00 & .02 & .322 \\
\hline Tentative & 4.10 & 2.01 & $3.36-4.84$ & 4.13 & 1.91 & $3.47-4.79$ & 0.00 & .00 & .953 \\
\hline Certainty & 0.48 & 0.47 & $0.31-0.65$ & 0.50 & 0.46 & $0.34-0.66$ & 0.04 & .00 & .843 \\
\hline \multicolumn{10}{|l|}{ Physical State } \\
\hline Body & 0.36 & 0.47 & $0.19-0.53$ & 0.21 & 0.25 & $0.12-0.30$ & 2.24 & .04 & .140 \\
\hline Sexuality & 0.01 & 0.07 & $0.02-0.04$ & 0.01 & 0.06 & $-0.01-0.31$ & 0.01 & .00 & .910 \\
\hline Eating & 0.11 & 0.19 & $0.04-0.18$ & 0.09 & 0.17 & $0.03-0.15$ & 0.11 & .00 & .746 \\
\hline Sleeping & 0.51 & 0.53 & $0.31-0.71$ & 0.43 & 0.45 & $0.27-0.59$ & 0.36 & .01 & .551 \\
\hline Grooming & 0.11 & 0.22 & $0.03-0.19$ & 0.08 & 0.16 & $0.03-0.14$ & 0.42 & .01 & .521 \\
\hline
\end{tabular}

